\title{
Evaluation of thermostable catalase-peroxidase AfKatG supplementation on toxicity of residual hydrogen peroxide in cultivation media of lactic acid bacteria from starter cultures
}

\author{
Eva Struhárňanská ${ }^{1}$, Monika Chovanová ${ }^{1}$, Silvia Rybecká ${ }^{1}$, Mária Mikulášová ${ }^{1}$, Zdenko \\ Levarski ${ }^{1,2}$, Marcel Zámocký ${ }^{3}$, Stanislav Stuchlík ${ }^{1,2}$ and Ján Turňa ${ }^{1}$ \\ ${ }^{1}$ Department of Molecular Biology, Faculty of Natural Sciences, Comenius University in Bratislava, Bratislava, Slovakia \\ ${ }^{2}$ Comenius University Science Park, Bratislava, Slovakia \\ ${ }^{3}$ Institute of Molecular Biology, Slovak Academy of Sciences, Bratislava, Slovakia
}

\begin{abstract}
Lactic acid bacteria (LAB) are exceptionally important strains in food industry. It is a heterogeneous group sharing same metabolic and physiological properties. They are usually catalase-negative strains, which represents a big disadvantage in food production in comparison with pathogenic bacteria as staphylococci and listeria existing in the same environment, because of the use of hydrogen peroxide as a disinfection agent which is utilized by catalases. We focused on increase in LAB surviving through the disinfection without any positive effect on growth of pathogenic bacteria. In our functional test hydrogen peroxide was used for disinfection. Ten mM thermostable catalase-peroxidase AfKat $G$ was added to solid media to cultivate bacteria afterwards. As predicted there was no difference in the growth of pathogenic bacteria with or without catalase-peroxidase addition to media. However, we showed a huge positive effect on surviving LAB. With addition of AfKatG to solid media we gained 2-38 times higher CFU/ml than in control samples without it. We can assume AfKatG as an excellent supplement for growth media of food strains.
\end{abstract}

Key words: Lactic acid bacteria - Thermostable catalase-peroxidase - Disinfection - Food pathogens

\section{Introduction}

Aerobic bacteria utilizing molecular oxygen for respiration and oxidation of nutrients to obtain energy have to cope with reactive oxygen species (ROS), e.g. $\mathrm{H}_{2} \mathrm{O}_{2}$, superoxide anion and hydroxyl radical, produced as incomplete reduction products of molecular oxygen (Cadenas 1989; Halliwell 1990). ROS are essential in a variety of different physiological functions but shifting the balance in favour of ROS results in cell oxidative stress (De Oru'e Lucana et al. 2012). Exposure to oxidative stress causes damage to various macromolecules, causing mutations and often cell death. Hydrogen peroxide works by producing destructive hydroxyl free radicals that

Correspondence to: Eva Struhárňanská, Department of Molecular Biology, Faculty of Natural Sciences, Comenius University, Ilkovičova 6, 84215 Bratislava, Slovakia

E-mail: struharnans1@uniba.sk can attack membrane lipids, DNA, and other essential cell components. Catalase may be the most important enzyme in protection against high concentrations of $\mathrm{H}_{2} \mathrm{O}_{2}$ for many kinds of bacteria because of their high turnover number in comparison to other systems involved in protection against $\mathrm{H}_{2} \mathrm{O}_{2}$ (George 1949; Jones and Suggett 1968; Jamieson et al.1986). Catalase can protect cells against hydrogen peroxide by degrading it to water and oxygen. Hydrogen peroxide is widely used as a disinfectant in food industry, ussually with concentration of 3-6\%.

Lactic acid bacteria (LAB) represent a heterogeneous group of gram-positive bacteria that are acid tolerant, generally nonsporulating, microaerophilic bacilli or cocci, which share the same metabolic and physiological properties, including the ability to degrade carbohydrates and produce lactic acid (Orla-Jensen 1919; Stiles and Holzapfel 1997; Klein et al. 1998). There are many genera in this group of bacteria, such as Oenococcus spp., Sporolactobacillus spp., 
Teragenococcus spp., Vagococcus spp., Lactobacillus spp., Leuconostoc spp., Pediococcus spp., Lactococcus spp., and Streptococcus spp., as well as Aerococcus spp., Carnobacterium spp., Enterococcus spp. and Weisella spp. (Vandamme et al. 2014). LAB are generally regarded as safe (GRAS), which only increases their industrial significance due to their ubiquitous occurrence in foods and their contribution to the necessary healthy human microbiota in the mucous membranes. They are among the most important groups of microorganisms used in the food industry, so it is important to find mechanisms to increase their viability.

Listeria monocytogenes is a gram-positive disease-causing bacterium in a wide variety of animal species, including mammals, birds, fish, and crustaceans. Due to its high ability to survive at low temperatures and to multiply even at $4^{\circ} \mathrm{C}$ it can contaminate food operations. Humans are most likely to be infected through consumption of contaminated foods and/or feedstuffs (Cartwright et al.2013). Listeria sp. are the most virulent alimentary pathogens.

Until recently, Staphylococcus epidermidis was considered a harmless commensal microorganism on human skin but it is currently considered a significant opportunistic pathogen. It is a common cause of nosocomial infections, about the same as its more virulent related Staphylococcus aureus (NNIS system report, 1992-2004). Staphylococcus epidermidis does not form spores but can cause contamination of food products during food preparation and processing.

AfKatG is a thermostable catalase-peroxidase with origin in archaeon Archaeoglobus fulgidus (Kengen et al. 2001) which we successfully produce heterologously in our laboratory (data not published). It is a dimeric protein with molecular weight of $169000 \mathrm{Da}$. The temperature optima of its catalase and peroxidase activity are $70^{\circ} \mathrm{C}$ and $80^{\circ} \mathrm{C}$, respectively (Kengen et al. 2001).

\section{Materials and Methods}

\section{Strains used}

This study includes a total of 21 strains of lactic acid bacteria (Table 1), 16 food strains of Staphylococcus epidermidis, and 16 strains of Listeria monocytogenes (Table 2) isolated from food-associated environments, mostly dairy processing plants.

\section{Media used for cultivation}

Brain heart infusion (BHI) broth (Biolife, Italy) was used for cultivation of Staphylococcus sp. strains. Tryptic soy broth (Biolife, Italy) was used for cultivation of Listeria sp. strains. MRS medium (Himedia, India) was used for cultivation of LAB. For solid media any broth was supplemented with $1.6 \%$ agar.

\section{Functional study of bacterial survival}

To monitor the impact of thermostable catalase-peroxidase on the survival of LAB after treatment with hydrogen peroxide the inhibition test was used. The overnight cultures of tested organisms were prepared under optimal conditions $\left(30^{\circ} \mathrm{C}\right.$ and microaerophilic environment for $\mathrm{LAB}, 37^{\circ} \mathrm{C}$ and aeration for staphylococci and listeria). The overnight cultures were used as inoculum in fresh media with ratio 1:100. Prepared suspensions were incubated until reaching $\mathrm{OD}_{600}=0.5$ (exponential growth phase), when we added hydrogen peroxide in the final concentration $10 \mathrm{mM}$ (experimentally determined concentration efficient for $2 \log _{10}$ reduction which represents $99 \%$ reduction of viable cells) and incubated for an additional hour. We chose exponential growth phase because of cell vitality and viability, thus the changes in growth are mostly due to external conditions and not biochemical processes like in overnight cultures. The number of surviving bacteria was determined by viable counts on Petri plates. Two sets of agar plates were used, the control ones without addition of AfKatG and test plates with addition of catalase-peroxidase in the final concentration of $10 \mathrm{mM}$. Each strain was used in three dilution series

Table 1. LAB strains used in work and fold of survival after thermostable catalase-peroxidase AfKatG addition to the solid media of LAB strains

\begin{tabular}{lcc}
\hline Species & Strain & Fold of survival \\
\hline Lactobacillus paraplantarum & 599 & $1.1 \pm 0.03$ \\
\hline Lactobacillus plantarum & 621 & $2.2 \pm 0.04$ \\
& 619 & $2.3 \pm 0.04$ \\
& 614 & $1.9 \pm 0.03$ \\
Lactobacillus brevis & 618 & $1.4 \pm 0.02$ \\
& 597 & $2.5 \pm 0.05$ \\
\hline & 613 & $9.0 \pm 0.22$ \\
Lactobacillus paracasei & 615 & $1.1 \pm 0.04$ \\
\hline Lactobacillus fermentum & 616 & $1.9 \pm 0.04$ \\
\hline Lactobacillus casei & 622 & $2.8 \pm 0.05$ \\
\hline Lactobacillus lactis & 623 & $1.3 \pm 0.02$ \\
\hline & 612 & $38.6 \pm 0.83$ \\
Leuconostoc mesenteroides & 620 & $3.3 \pm 0.12$ \\
\hline Leuconostoc pseudomesenteroides & 598 & $12.7 \pm 0.26$ \\
\hline Enterococcus durans & 17 & $5.3 \pm 0.13$ \\
\hline Enterococcus sp. & 610 & $3.3 \pm 0.07$ \\
\hline
\end{tabular}

* not able to calculate because of lack of bacteria growth without catalase addition. 
and three independent biological tests. Data are presented as mean values \pm standard deviation. The obtained results were evaluated as the ratio of cell survival enhancement to the addition of catalase-peroxidase to culture cells media compared to control samples and statistical significance was calculated using Student's $t$-test.

\section{Results}

Effect of AfKat $G$ addition to growth media of lactic acid bacteria

The positive effect of addition of thermostable AfKatG to growth solid media of lactic acid bacteria was statistically significant ( $p=0.00084$ ) in comparison to control samples. Direct functional evidence of the effect of AfKatG addition on cell viability is their increased survival. Of the 21 strains tested, 13 grew at least twice CFU (colony forming unit)/ml with the addition of AfKatG, while five strains grew more than 5 times the $\mathrm{CFU} / \mathrm{ml}$ than colonies in control samples (Table 1). Not only genus but also species dependence effect of the addition of catalase-peroxidase to the media were observed (Table 1).

\section{Effect of AfKat G addition to growth media of lactobacilli}

There were two groups of Lactobacillus spp. - fast (2-3 days) and slow (4 days) growing. Although L. paraplantarum (599) and L. plantarum $(597,614,618,619,621)$ grow relatively

Table 2. Food strains of Listeria monocytogenes used in work

\begin{tabular}{lcc}
\hline Strain & Serotype & Persistence \\
\hline P1 & 1IIc & Y \\
P2 & $2 \mathrm{IIa}$ & $\mathrm{Y}$ \\
P3 & $9 \mathrm{IVb}$ & $\mathrm{Y}$ \\
P4 & $2 \mathrm{IIa}$ & CCM strain \\
P30 & $1 \mathrm{IIc}$ & $\mathrm{Y}$ \\
P44 & $13 \mathrm{IIa}$ & $\mathrm{N}$ \\
P76 & $1 \mathrm{IIc}$ & $\mathrm{Y}$ \\
$\mathrm{N} 6$ & $6 \mathrm{IIa}$ & $\mathrm{N}$ \\
$\mathrm{N} 12$ & $15 \mathrm{IIb}$ & $\mathrm{N}$ \\
$\mathrm{N} 17$ & $17 \mathrm{IVb}$ & $\mathrm{N}$ \\
$\mathrm{N} 19$ & $18 \mathrm{IIa}$ & $\mathrm{N}$ \\
$\mathrm{N} 22$ & $21 \mathrm{IVb}$ & $\mathrm{N}$ \\
$\mathrm{N} 1$ & $3 \mathrm{IIa}$ & $\mathrm{N}$ \\
$\mathrm{N} 2$ & $4 \mathrm{IIa}$ & $\mathrm{N}$ \\
P57 & $2 \mathrm{IIa}$ & $\mathrm{Y}$ \\
P71 & $9 \mathrm{IVb}$ & $\mathrm{Y}$ \\
\hline $\mathrm{Y}$ & &
\end{tabular}

Y, yes; N, no. rapidly under optimal conditions $\left(28-30^{\circ} \mathrm{C}\right)$, and formed a large number of colonies, the addition of catalase did not have the essential effect on cell survival (Figure 1A). In slow-growing species, the differences were much more pronounced, despite the overall lower CFU/ml. The effect on L. brevis strains was dual, either there was significantly increased the cells ability to grow (up to 9-fold) in the 613 strain, or there was no effect at all with strain 615 (Figure 1B). The addition of catalase had no significant effect on L. paracasei strains $(616,622,623)$. The most pronounced survival efficacy of catalase was observed in L. fermentum 612 , where cell survival was 38.6 times greater than in control samples. There was also observed a significant effect of the catalase addition on the cell viability in L. casei (12.7-fold) and L. lactis (5.3-fold) after treatment with hydrogen peroxide (Figure 1B).

\section{Effect of AfKatG addition to growth media of Leuconostoc spp. and Enterococcus spp. strains}

The apparent effect of catalase-peroxidase addition to media on the growth of Leuconostoc spp. strains was observed while being less effective only with strain 608 . However, in two strains, 609 and 610, the difference in cell survival was very pronounced (Figure 1C). Strain 611 could not be included to the fold survival as no colonies formed without the addition of AfKatG to the culture media (Table 1) and thus no cells survived hydrogen peroxide treatment, whereas after AfKatG addition there were about $6 \log _{10} \mathrm{CFU} / \mathrm{ml}$.

The genus Enterococcus spp. has also proven to be very susceptible to the hydrogen peroxide treatment (Figure 1D). After the addition of AfKatG to the culture media, the increase in the CFU/ml was observed. Strain 617 reached 1.8 times more colonies. There was a more pronounced difference in strain 624 with 9.4 times more colonies.

\section{Effect of AfKat $G$ addition to growth media of food pathogenic bacteria}

The food opportunistic and obligate pathogens found in the same environment include Staphylococcus epidermidis and Listeria monocytogenes. The addition of AfKatG to the media proved no effect to the growth of bacteria. No significant difference ( $p=0.1631)$ was noticed between control samples and samples with the addition of AfKatG, whether in listeria (Figure 1E) or staphylococci (Figure 1F).

\section{Discussion}

$\mathrm{LAB}$ are among the most important and most industrially used microorganisms (Sonomoto et al. 2011). They are important in starter cultures in food processing, but they 
do not usually possess catalase which may be a significant cultivation disadvantage compared to pathogens existing in the same environment (e.g. staphylococci and listeria). This study was focused on increasing survival ability of LAB during sanitation process with $\mathrm{H}_{2} \mathrm{O}_{2}$ as a well-known antiseptic in food industry (McDonnell and Russell 1999). A functional test, in which, after treatment with hydrogen peroxide the thermostable catalase-peroxidase AfKatG was added (pro- duced in our laboratory) to solid media, was performed. We found that this effect is not only genus-dependent but also species-dependent. Addition of AfKatG in any of the samples tested had no negative effects on cell growth. Of the $21 \mathrm{LAB}$ strains tested, 13 strains grew at least twice the CFU/ml with the addition of AfKatG, while five strains increased colony count almost 5 -fold (Table 1 ) and the addition proved to be statistically significant. The smallest effect (up to 2.5 times
A

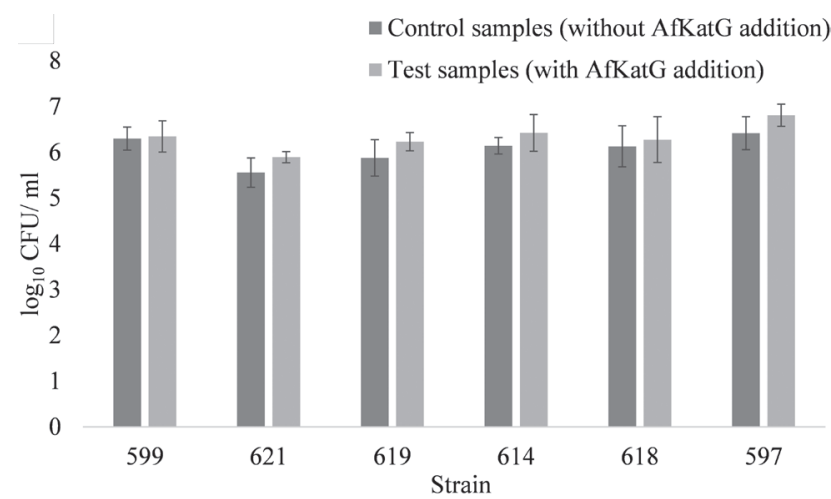

C

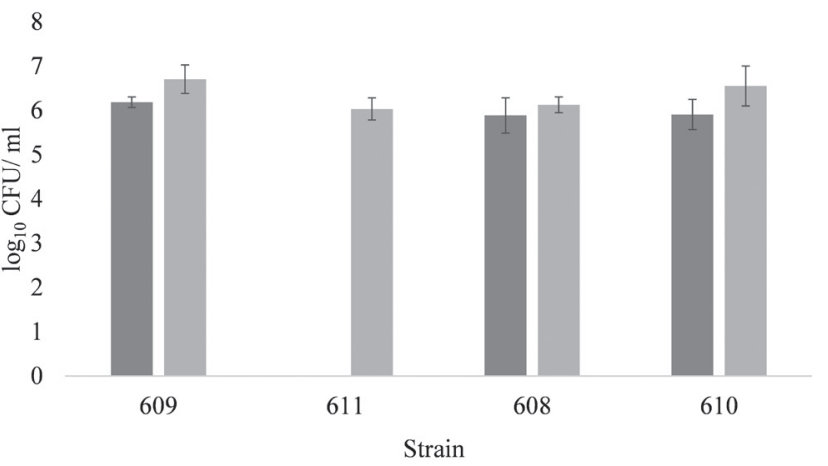

E

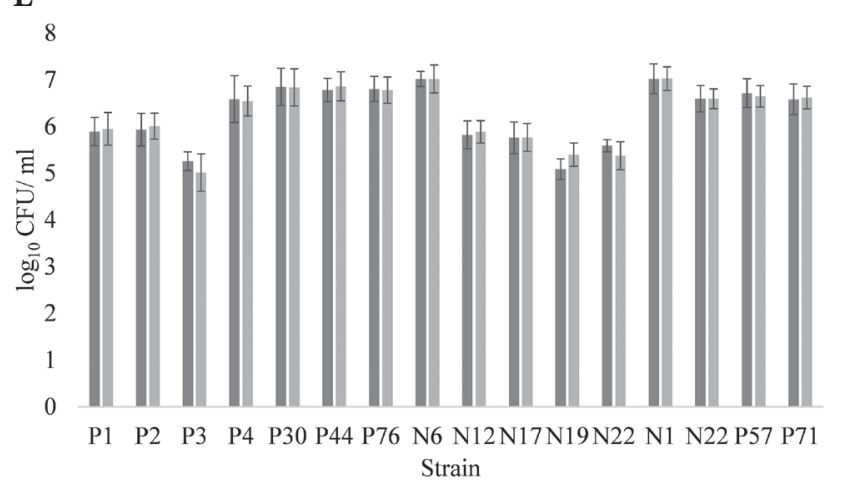

B

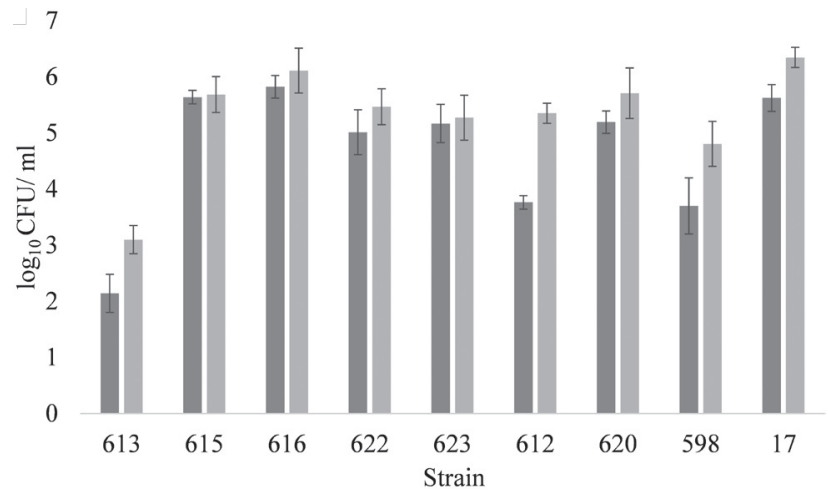

D

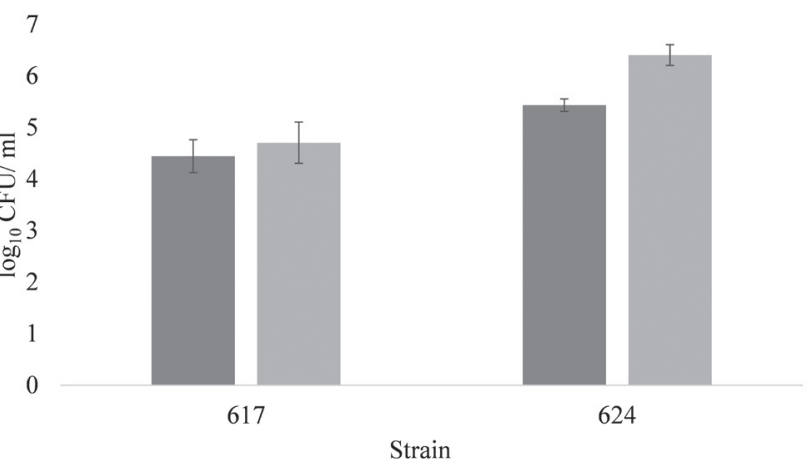

F

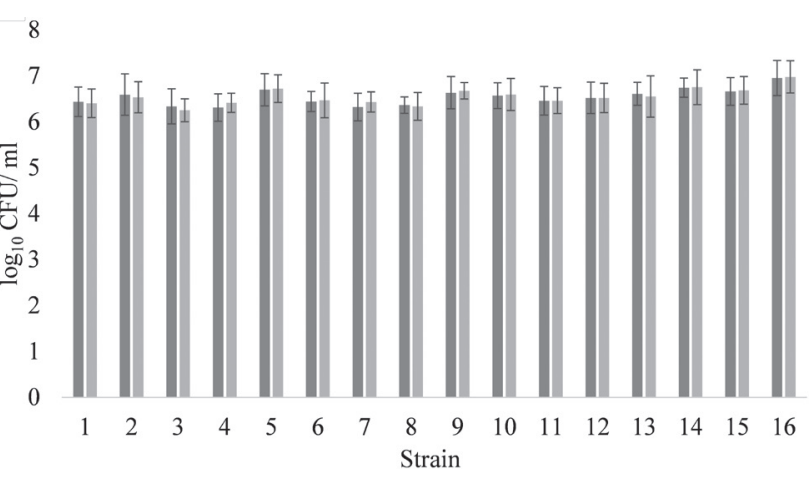

Figure 1. The effect of addition of thermostable AfKatG to growth solid media of Lactobacillus spp. (fast growing, A; slowly growing, B), Leuconostoc spp. (C), Enterococcus spp. (D), Listeria monocytogenes (E) and Staphylococcus epidermidis (F) strains after hydrogen peroxide treatment in comparison to control samples (without addition of AfKatG). CFU, colony forming unit. 
the CFU/ml compared to the control) was observed in strains L. paraplantarum and L. plantarum. It was found that starter culture of $L$. plantarum produced low concentrations of $\mathrm{H}_{2} \mathrm{O}_{2}$ (Raccach and Baker 1978) and thus can deal with certain level of exogenous $\mathrm{H}_{2} \mathrm{O}_{2}$. Not possessing heme, lactobacilli, as well as other lactic acid-producing bacteria, do not utilize the cytochrome system (which reduces oxygen to water) for terminal oxidation during their respiratory processes. It was also shown that they possess alkyl hydroperoxide reductase as another defence system against $\mathrm{H}_{2} \mathrm{O}_{2}$ (Archibald and Fridovich 1981). The other species of the genus Lactobacillus were more pronounced to the effect of adding AfKatG to the media, the greatest effect was found in L. fermentum, strain 612 when the addition of AfKatG increased cell survival by 38 -fold. Such an increase in the survival can be caused by being less naturally occurring and, moreover, growing slower than the L. plantarum and L. paraplantarum, making them more susceptible to ROS.

We did not support growth of pathogenic Staphylococcus epidermidis and Listeria monocytogenes strains. The effect was expected as these species are catalase positive. There was no statistical dependence on addition of AfKatG. We assume staphylococci and listeria strains as aerobic organisms are forced to break down ROS that develop through oxygen utilization. They possess more mechanisms to do that than LAB. Some of the ROS induce gene expression of enzymes involved in utilizing them. That means if there was any residual peroxide these organisms could break it down. LAB do not possess as many mechanisms as other tested strains but their dividing time is much longer and because of that AfKatG addition in solid media decompose any residual hydrogen peroxide. This effect only proves AfKatG as a perfect supplement for starter culture media, it increases survival of industrially important, yet has no effect on growth of pathogenic bacteria.

Our results suggest that introduction of AfKatG addition to cultivation media of either starter cultures during dairy processing or cultivation media used for detection and monitoring of microbial growth, can significantly enhance the growth of common LAB and desired industrial strains, while not promoting the growth of pathogenic species. AfKatG addition to starter cultures after disinfection could rapidly increase growth of $\mathrm{LAB}$ as the residual hydrogen peroxide would be broken down by it.

Acknowledgement. This research is result of implementation of projects „Synthetic biology and production of peroxidases de novo“ (under contract No. APVV-14-0375), „Preparation of natural flavors by biotransformation using the comprehensive analytical methods" (under contract No. APVV-15-0466), and "BIOREKPROT” (ITMS 26240220048) supported by the Research and Innovation Operational Programme funded by ERDF. We thank Dr. Tomáš Kuchta (Food Research Institute, National Agricultural and Food Centre, Slovakia) for providing us with all food strains tested in this work.

\section{References}

Archibald FS, Fridovich I (1981): Manganese and defenses against oxygen toxicity in Lactobacillus plantarum. J. Bacteriol. 145, $442-451$

Cadenas E (1989): Biochemistry of oxygen toxicity. Ann. Rev. Biochem. 58, 79-110

https://doi.org/10.1146/annurev.bi.58.070189.000455

Cartwright EJ, Jackson KA, Johnson SD, Graves LM, Silk BJ, Mahon BE (2013): Listeriosis outbreaks and associated food vehicles, United States, 1998-2008. Emerg. Infect. Dis. 19, 1-9 https://doi.org/10.3201/eid1901.120393

National Nosocomial Infections Surveillance (NNIS) System Report, data summary from January 1992 through June 2004, issued October 2004. Am. J. Infect. Control. 32, 470-485 https://doi.org/10.1016/j.ajic.2004.10.001

De Oru'e Lucana DO, Wedderhoff I, Groves MR (2012): ROSmediated signalling in bacteria: zinc-containing cys- $\mathrm{X}-\mathrm{x}-\mathrm{cys}$ redox centres and iron-based oxidative stress. J. Sig. Trans. 2012, 605905

https://doi.org/10.1155/2012/605905

George P (1949): The effect of the peroxide concentration and other factors on the decomposition of hydrogen peroxide by catalase. Biochem. J. 44, 197-205 https://doi.org/10.1042/bj0440197

Halliwell B (1990): How to characterize a biological antioxidant. Free Rad. Res. Commun. 9, 1-32 https://doi.org/10.3109/10715769009148569

Jamieson D, Chance B, Cadenas E, Boveris A (1986): The relation of free radical production to hyperoxia. Annu. Rev. Physiol. 48, 703-719

https://doi.org/10.1146/annurev.ph.48.030186.003415

Jones P, Suggett A (1968): The catalse-hydrogen peroxide system. Kinetics of catalatic action at high substrate concentrations. Biochem. J. 110, 617-620 https://doi.org/10.1042/bj1100617

Kengen SW, Bikker FJ, Hagen WR, Vos WM, Oost J (2001): Characterization of a catalase-peroxidase from the hyperthermophilic archaeon Archaeoglobus fulgidus. Extremophiles 5, 323-332 https://doi.org/10.1007/s007920100208

Klein G, Pack A, Bonaparte C, Reuter G (1998): Taxonomy and physiology of probiotic lactic acid bacteria. Int. J. Food Microbiol. 41, 103-125 https://doi.org/10.1016/S0168-1605(98)00049-X

McDonnell G, Russell AD (1999): Antiseptics and disinfectants: activity, action, and resistance. Clin. Microbiol. Rev. 12, 147-179 https://doi.org/10.1128/CMR.12.1.147

National Nosocomial Infections Surveillance (NNIS) System Report, data summary from January 1992 through June 2004, issued October 2004. Am. J. Infect. Control. 32, 470-485 https://doi.org/10.1016/j.ajic.2004.10.001

Orla-Jensen S (1919): The Lactic Acid Bacteria. Fred Host and Son, Copenhagen, Denmark

Raccach M, Baker RC (1978): Formation of hydrogen peroxide by meat starter cultures. J. Food Prot. 41, 798-799 https://doi.org/10.4315/0362-028X-41.10.798 
Sonomoto K, Yokota A (2011): Lactic Acid Bacteria and Bifidobacteria: Current Progress in Advanced Research. Caister Academic Press, Hokkaido, Japan

Stiles ME, Holzapfel WH (1997): Lactic acid bacteria of foods and their current taxonomy. Int. J. Food Microbiol. 36, 1-29 https://doi.org/10.1016/S0168-1605(96)01233-0
Vandamme P, De Bruyne K, Pot B (2014): Phylogenetics and systematics. In: Lactic Acid Bacteria: Biodiversity and Taxonomy. (Eds. Holzapfel WH, Wood BJB), pp 31-44, John Wiley \& Sons, Ltd, Chichester, United Kingdom https://doi.org/10.1002/9781118655252.ch3

Received: June 11, 2019

Final version accepted: July 30, 2019 